\title{
(-)-Epigallocatechin gallate inhibits the expression of indoleamine 2,3-dioxygenase in human colorectal cancer cells
}

\author{
KENGO OGAWA $^{1}$, TAKESHI HARA ${ }^{1}$, MASAHITO SHIMIZU ${ }^{1}$, JUNJI NAGANO ${ }^{1}$, \\ TOMOHIKO OHNO ${ }^{1}$, MASATO HOSHI ${ }^{2}$, HIROYASU ITO ${ }^{2}$, HISASHI TSURUMI ${ }^{1}$, \\ KUNIAKI SAITO $^{3}$, MITSURU SEISHIMA ${ }^{2}$ and HISATAKA MORIWAKI ${ }^{1}$ \\ Departments of ${ }^{1}$ Internal Medicine and ${ }^{2}$ Informative Clinical Medicine, Gifu University Graduate School of Medicine, \\ Gifu 501-1194; ${ }^{3}$ Human Health Sciences, Graduate School of Medicine and Faculty of Medicine, \\ Kyoto University, Kyoto 606-8501, Japan
}

Received March 5, 2012; Accepted June 8, 2012

DOI: $10.3892 / \mathrm{ol} .2012 .761$

\begin{abstract}
Immune escape, the ability of tumor cells to avoid tumor-specific immune responses, occurs during the development and progression of several types of human malignancies, including colorectal cancer (CRC). Indoleamine 2,3-dioxygenase (IDO), the tryptophan catabolic enzyme, plays a significant role in regulating the immune response and provides tumor cells with a potent tool to evade the immune system. In the present study, we examined the effects of (-)-epigallocatechin gallate (EGCG), the major catechin in green tea, on the inhibition of IDO expression induced by interferon (IFN)- $\gamma$ in human CRC cells. We found that IFN- $\gamma$ increased the expression levels of IDO protein and mRNA in HT29 and SW837 CRC cell lines. Treatment of SW837 cells with EGCG significantly decreased IFN- $\gamma$-induced expression of IDO protein and mRNA in a dose-dependent manner. Enzymatic activity of IDO, determined by the concentration of L-kynurenine in the culture medium, was also significantly inhibited by EGCG treatment. Phosphorylation of signal transducer and activator of transcription 1 (STAT1) induced by IFN $-\gamma$ was also significantly inhibited by EGCG. Reporter assays indicated that EGCG inhibited the transcriptional activities of IDO promoters, IFN-stimulated response element and
\end{abstract}

Correspondence to: Dr Masahito Shimizu, Department of Internal Medicine, Gifu University Graduate School of Medicine, 1-1 Yanagido, Gifu 501-1194, Japan

E-mail: shimim-gif@umin.ac.jp

Abbreviations: CRC, colorectal cancer; EGCG, (-)-epigallocatechin gallate; GAS, interferon- $\gamma$ activation sequence; IDO, indoleamine 2,3-dioxygenase; IFN, interferon; ISRE, interferon-stimulated response element; JAK, janus kinase; RT-PCR, reverse transcription-polymerase chain reaction; STAT1, signal transducer and activator of transcription 1

Key words: indoleamine 2,3-dioxygenase, (-)-epigallocatechin gallate, colorectal cancer cells, interferon- $\gamma$, signal transducer and activator of transcription 1
IFN- $\gamma$ activation sequence, activated by STAT1 phosphorylation. These findings suggest that EGCG may exert antitumor effects on CRC, at least in part, by inhibiting the expression and function of IDO through the suppression of STAT1 activation. EGCG may, thus, serve as a potential agent for antitumor immunotherapy and be useful in the chemoprevention and/or treatment of CRC.

\section{Introduction}

Numerous immune effector cells and molecules recognize and destroy preneoplastic cells (1). The escape of such cells from the immune system is involved in the development and progression of several types of tumors (2). Recent studies have suggested that tryptophan catabolism, via indoleamine 2,3-dioxygenase (IDO), may be a critical mechanism of immune escape (3). IDO, an intracellular enzyme that degrades the essential amino acid tryptophan along the kynurenine pathway, is constitutively expressed by tumor cells and dendritic cells in tumor-draining lymph nodes (4). L-kynurenine and certain other metabolites derived from tryptophan by IDO may inhibit proliferation and induce apoptosis in $\mathrm{T}$ cells and natural killer cells (5). IDO overexpression correlates with poor clinical outcomes in patients with several types of malignancies, including colorectal cancer (CRC) (6-8). We have recently demonstrated that increased levels of IDO expression in tumor cells and serum concentration of L-kynurenine, which reflects the enzymatic activity of IDO, are associated with poor prognosis in patients with diffuse large B-cell lymphoma (9-11). Alternatively, 1-methyl-tryptophan, an IDO inhibitor, effectively suppresses chemically induced colorectal carcinogenesis in rats (12). These studies suggest that targeting IDO and regulating tryptophan catabolism may be effective strategies for the treatment of certain types of human malignancies, including CRC (6).

Several immune factors are regarded as mediators in the regulation of IDO expression. Among these factors, interferon (IFN)- $\gamma$, which is released by activated $\mathrm{T}$ cells and natural killer cells within the tumor microenvironment (13), is considered to be a major inducer of IDO in numerous human 
cell types (14). IFN- $\gamma$ binding to its receptor results in the phosphorylation of janus kinase (JAK), which phosphorylates the downstream protein signal transducer and activator of transcription 1 (STAT1) (13). The IDO promoter contains multiple sequence elements that confer responsiveness to IFN- $\gamma$, including the interferon-stimulated response element (ISRE) and the IFN- $\gamma$ activation sequence (GAS) (14). IFN- $\gamma$ induces IDO by promoting ISRE and GAS sequence elements through the activation of the JAK/STAT1 signaling pathway (15), indicating that the inhibition of STAT1 phosphorylation and its downstream promoter activity may be effective for the downregulation of IDO (16).

(-)-Epigallocatechin gallate (EGCG), the major biologically active component of green tea, exerts its anticancer and cancer chemopreventive effects in various organs. These effects are partially attributed to their antioxidative, antiangiogenic and antimutagenic effects, as well as their anti-inflammatory activities $(17,18)$. We previously demonstrated that EGCG is able to suppress cell proliferation and induce apoptosis in human CRC cells (19-21). The inhibitory effects of EGCG on inflammation- and obesity-related colon carcinogenesis have also been demonstrated in rodent models $(22,23)$. In addition, recent studies revealed that EGCG treatment suppresses the expression of IDO stimulated by IFN- $\gamma$ in murine dendritic cells (24) as well as in various human cancer-derived cell lines $(25,26)$. These studies suggest that the inhibitory effect of EGCG on IDO expression may contribute to the chemopreventive and anticancer properties of EGCG; however, whether EGCG is able to inhibit expression of IDO in human CRC cells has not yet been examined. The present study investigated the effects of EGCG on the induction of IDO in human CRC cells stimulated with IFN- $\gamma$.

\section{Materials and methods}

Chemicals. EGCG was obtained from Mitsui Norin Co. (Tokyo, Japan) and recombinant human IFN- $\gamma$ was purchased from PeproTech (Rocky Hill, NJ, USA).

Cell lines and cell culture. The Caco2, HCT116, HT29, SW480 and SW837 human CRC cell lines were obtained from the American Type Culture Collection (Manassas, VA, USA). All cell lines were maintained in DF10 medium containing Dulbecco's modified Eagle's medium (DMEM; Life Technologies, Grand Island, NY, USA) supplemented with $10 \%$ fetal bovine serum (FBS). Cells were then cultured in a humidified incubator with $5 \% \mathrm{CO}_{2}$ at $37^{\circ} \mathrm{C}$. The experimental protocol of this study was approved by the Ethics Committee of Gifu University.

RNA extraction and quantitative real-time reverse transcription-polymerase chain reaction $(R T-P C R)$ analysis. Total RNA was extracted using the RNeasy Mini kit (Qiagen, Valencia, CA, USA). Quantitative real-time RT-PCR was performed on $1 \mu \mathrm{g}$ total RNA using specific primer/probe sets that amplify IDO and glyceraldehyde 3-phosphate dehydrogenase (GAPDH) genes (TaqMan Gene Expression Assays; Life Technologies) and Toyobo Real-time PCR Master mix (Toyobo, Osaka, Japan). Each sample was analyzed on a LightCycler 1.0 (Roche Diagnostics GmbH, Mannheim,
Germany) (27). Gene expression levels were normalized to GAPDH expression levels using a standard curve.

Protein extraction and western blot analysis. Total cellular protein was extracted and equivalent amounts of protein $(20 \mu \mathrm{g} /$ lane $)$ were examined by western blot analysis $(21,28)$. The primary antibody for IDO was purchased from Abcam (Cambridge, UK). The primary antibodies for STAT1, phospho-STAT1 (tyr701) and GAPDH were purchased from Cell Signaling Technology (Danvers, MA, USA). An antibody to GAPDH was used as a loading control.

Determination of L-kynurenine concentration. Culture medium was deproteinized with 2 volumes of $3 \%$ perchloric acid. Following centrifugation, the concentration of L-kynurenine in the supernatants was measured by high-performance liquid chromatography (29).

ISRE and GAS reporter assays. Reporter assays were performed (20). ISRE and GAS luciferase reporter plasmids were purchased from Qiagen. Plasmid DNA $(0.1 \mu \mathrm{g})$ was transfected into SW837 cells $\left(3 \times 10^{4}\right.$ cells $/ 6.35 \mathrm{~mm}$ diameter dish) using Lipofectin reagent (Life Technologies) in Opti-MEM I medium (Life Technologies). After $22 \mathrm{~h}$, cells were preincubated in the absence or presence of various EGCG concentrations $(10,50$ or $100 \mu \mathrm{M})$ for $2 \mathrm{~h}$ and then stimulated with IFN- $\gamma(10 \mathrm{ng} / \mathrm{ml})$ for $24 \mathrm{~h}$. Cell extracts were prepared and luciferase activity was measured using a luciferase assay system (Promega, Madison, WI, USA). Cells were cotransfected with a CMV- $\beta$-galactosidase reporter in all reporter assays and differences in transfection efficacy were corrected by normalizing the luciferase activities to the $\beta$-galactosidase activities (20).

Statistical analysis. All data were expressed as the mean \pm standard deviation (SD). Statistical significance was evaluated using the Tukey-Kramer multiple comparison test and $\mathrm{P}<0.05$ was considered to indicate a statistically significant difference.

\section{Results}

Expression levels of IDO in human CRC cells in the presence and absence of IFN- $\gamma$ stimulation. We initially examined the expression levels of IDO in the presence and absence of IFN- $\gamma$ in Caco2, HCT116, HT29, SW480 and SW837 human CRC cells. Quantitative real-time RT-PCR analysis revealed that IDO mRNA was not expressed in any of the CRC cell lines without IFN- $\gamma$ stimulation. However, when the cell lines were stimulated with $10 \mathrm{ng} / \mathrm{ml} \mathrm{IFN}-\gamma$ for $24 \mathrm{~h}$, IDO mRNA levels were significantly increased in all cell lines, particularly SW837 and HT29 cells (Fig. 1A). Furthermore, in accordance with the expression levels of mRNA, the cellular levels of IDO protein in the SW837 and HT29 cells were markedly increased by IFN- $\gamma$ stimulation (Fig. 1B), indicating that CRC cells may induce IDO expression following IFN- $\gamma$ stimulation. Based on these results, SW837 cells were selected for the following experiments.

Effects of EGCG on the expression levels of IDO in SW837 CRC cells. We next examined the effects of EGCG on the 

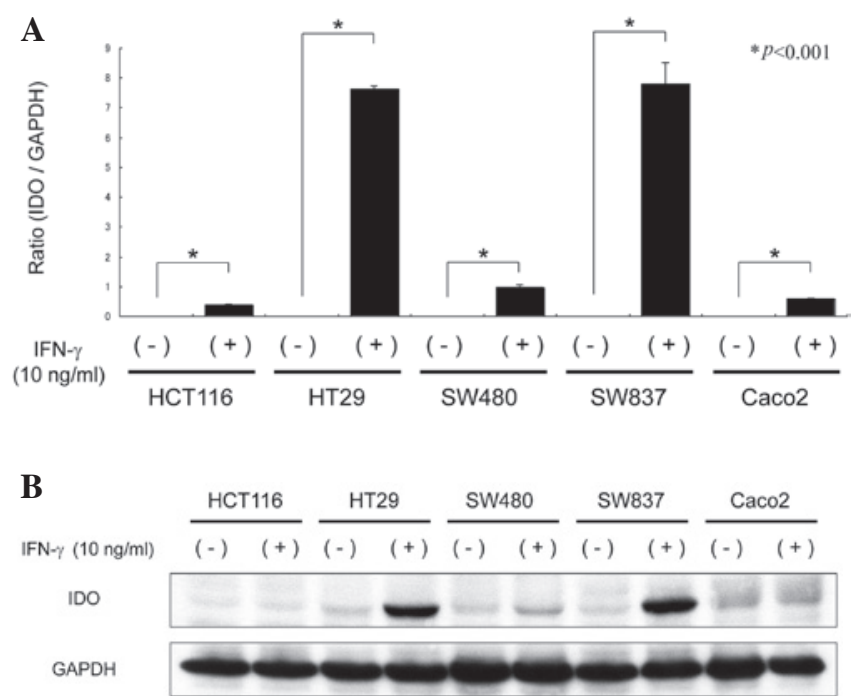

Figure 1. Expression of IDO in human CRC cell lines. Caco2, HCT116, HT29, SW480 and SW837 cells were treated with or without IFN- $\gamma(10 \mathrm{ng} / \mathrm{ml})$ for 24 h. (A) Total RNA was extracted from each cell line and IDO mRNA expression was measured by quantitative real-time RT-PCR. Gene expression levels were normalized to GAPDH. Columns and bars indicate the mean and SD. (B) IDO protein expression levels were detected by western blot analysis. Total cellular protein was extracted and equivalent amounts of protein were examined. Equal protein loading was confirmed by the detection of GAPDH. Repeated western blots gave similar results. IDO, indoleamine 2,3-dioxygenase; GAPDH, glyceraldehyde 3-phosphate dehydrogenase; IFN, interferon; RT-PCR, reverse transcription-polymerase chain reaction; $\mathrm{SD}$, standard deviation.

expression of IDO mRNA and protein in SW837 cells. As demonstrated in Fig. 2A, expression levels of IDO mRNA induced by IFN- $\gamma(\mathrm{P}<0.001)$ were significantly suppressed when the cells were pretreated with 50 and $100 \mu \mathrm{M}$ EGCG for $2 \mathrm{~h}$ prior to IFN- $\gamma$ stimulation for $24 \mathrm{~h}(\mathrm{P}<0.001$ for each comparison). Similarly, increasing levels of EGCG treatment, particularly $100 \mu \mathrm{M}$, caused a marked decrease in the levels of IDO protein induced by IFN- $\gamma$ in SW837 cells (Fig. 2B). These findings suggest that EGCG inhibits the expression of IDO mRNA and protein levels.

Effects of EGCG on the enzymatic activity of IDO induced by IFN- $\gamma$ in SW837 cells. We examined whether EGCG inhibits the enzymatic activity of IDO induced by IFN- $\gamma$ in SW837 cells. IDO activity was determined by measuring the concentration of L-kynurenine in culture medium (29). As demonstrated in Fig. 2C, the L-kynurenine concentration was markedly increased in the culture medium of IFN- $\gamma$-stimulated SW837 cells compared to that of the untreated cells $(\mathrm{P}<0.001)$. This increase was significantly inhibited by treatment with $100 \mu \mathrm{M}$ EGCG $(\mathrm{P}<0.001)$, suggesting that, in addition to the expression levels, EGCG also suppresses the enzymatic activity of IFN- $\gamma$-induced IDO in CRC cells.

Effects of EGCG on the phosphorylation of STAT1 protein in SW837 cells. To determine the molecular mechanisms involved in the EGCG suppression of IDO expression in CRC cells, we examined whether EGCG inhibits STAT1 protein phosphorylation as the JAK/STAT1 pathway is medi-
A
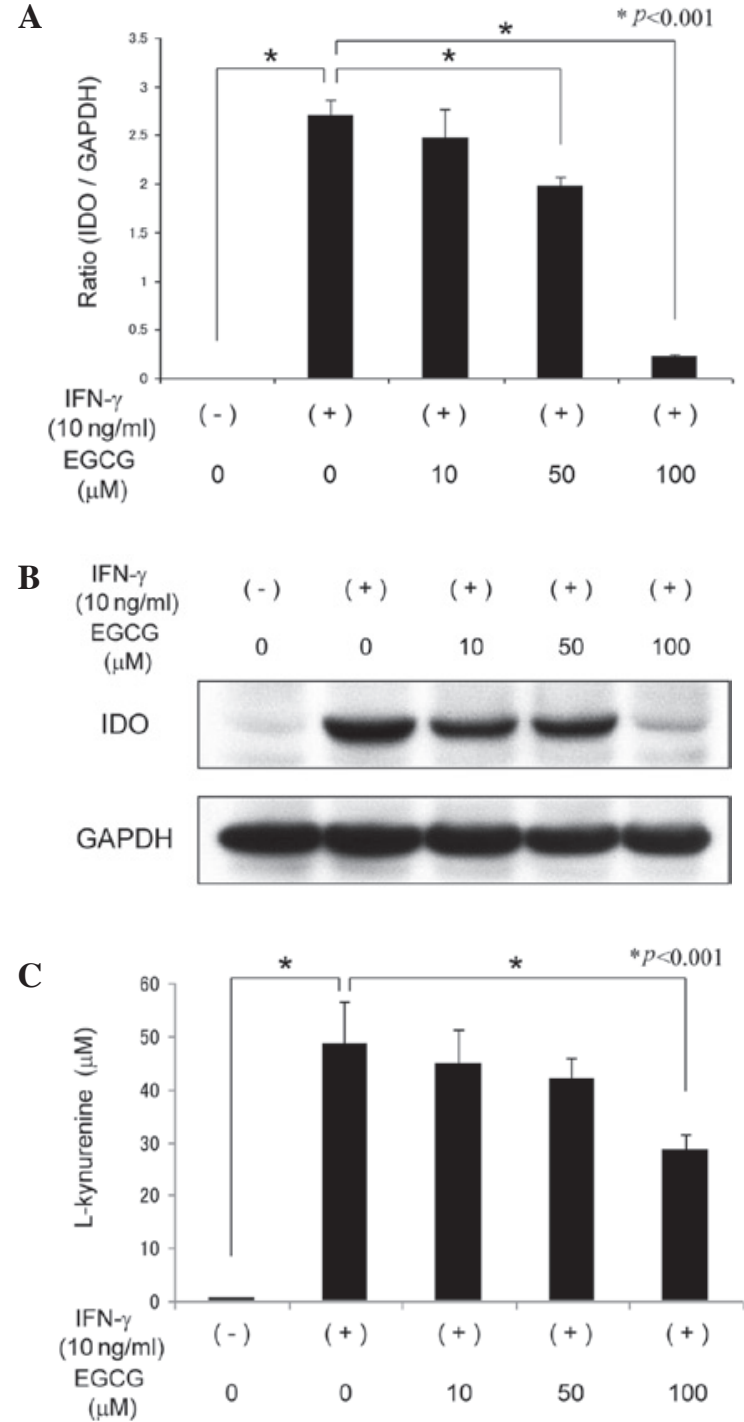

Figure 2. Effects of EGCG on the expression levels and enzymatic activity of IDO in SW837 cells. Cells were pretreated with various concentrations $(0,10,50$ or $100 \mu \mathrm{M})$ of EGCG for $2 \mathrm{~h}$ and were then stimulated with IFN- $\gamma$ for $24 \mathrm{~h}$. (A) Total RNA was extracted and the IDO mRNA expression levels were measured by quantitative real-time RT-PCR. (B) Total cellular protein was extracted and the levels of IDO protein expression were determined by western blot analysis. (C) Functional IDO enzymatic activity was determined by measuring the concentrations of L-kynurenine in culture medium under the same conditions. Columns and bars indicate the mean and SD. Repeated western blots gave similar results. IDO, indoleamine 2,3-dioxygenase; GAPDH, glyceraldehyde 3-phosphate dehydrogenase; IFN, interferon; EGCG, (-)-epigallocatechin gallate; RT-PCR, reverse transcription-polymerase chain reaction; SD, standard deviation.

ated by IFN- $\gamma$ to induce IDO expression (14). Western blot analysis revealed that stimulation with IFN- $\gamma$ for $30 \mathrm{~min}$ markedly increased the expression of phosphorylated STAT1 protein in SW837 cells. However, this induction was significantly decreased by EGCG treatment in a dose-dependent manner (Fig. 3A), suggesting that at least part of the EGCG inhibition of the IFN- $\gamma$-induced IDO expression was due to the suppressed activation of STAT1.

Effects of EGCG on the transcriptional activity of IDO promoters in SW837 cells. To elucidate whether transcription 

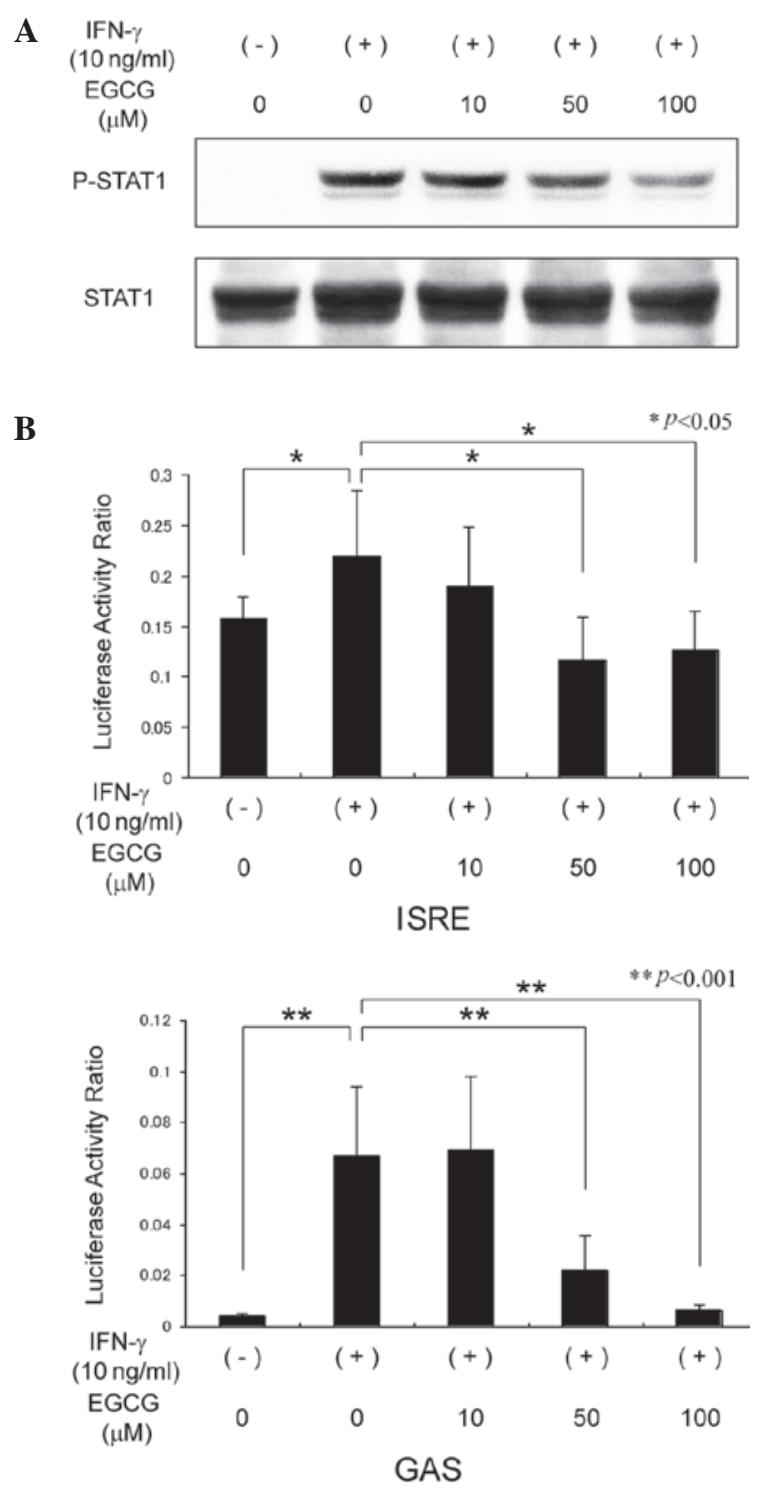

Figure 3. Effects of EGCG on the phosphorylation of STAT1 protein and transcriptional activity of IDO promoter in SW837 cells. (A) Cells were pretreated with various concentrations of EGCG for $2 \mathrm{~h}$ and were then stimulated with IFN- $\gamma$ for $30 \mathrm{~min}$. Total cellular protein was extracted and the expression of phosphorylated STAT1 protein was determined by western blot analysis. (B) Transient transfection reporter assays using ISRE and GAS luciferase reporter plasmids were performed using cell extracts pretreated with various concentrations of EGCG for $2 \mathrm{~h}$ and then stimulated with IFN- $\gamma$ for $24 \mathrm{~h}$. Columns and bars indicate the mean and SD. IFN, interferon; EGCG, (-)-epigallocatechin gallate; STAT1 signal transducer and activator of transcription 1; ISRE, interferon-stimulated response element; GAS, IFN- $\gamma$ activation sequence; IDO, indoleamine 2,3-dioxygenase; SD, standard deviation.

of IDO is inhibited by EGCG, we next examined the effects of EGCG on the transcriptional activity of ISRE and GAS, two sequence elements of the IDO promoter, which are activated by the binding of STAT1 and associated with IFN- $\gamma$-induced IDO gene transcription (15). Transient transfection luciferase reporter assays demonstrated that IFN $-\gamma$ stimulation upregulated the transcriptional activity of ISRE $(\mathrm{P}<0.05)$ and GAS $(\mathrm{P}<0.001)$ in SW837 cells. However, treatment with 50 and $100 \mu \mathrm{M}$ EGCG significantly inhibited the promoter activities of ISRE $(\mathrm{P}<0.05)$ and GAS $(\mathrm{P}<0.001)$ induced by IFN- $\gamma$ (Fig. 3B). These results provide evidence that the inhibitory effects of EGCG on IDO expression occur at the gene transcription level.

\section{Discussion}

The IDO-mediated escape of cancer cells from the immune system plays a critical role in tumor development and progression. Therefore, inhibition of inappropriate IDO activity in tumors may attenuate the ability of malignant cells to evade immune surveillance and promote their clearance (2). Several preclinical studies have demonstrated that IDO inhibitors, including 1-methyl-tryptophan, have a therapeutic effect in rodent cancer models $(30,31)$. The results of this study in human CRC cells provide the first evidence that a naturally occurring compound, EGCG, effectively inhibits the IFN- $\gamma$-induced expression and activity of IDO (Fig. 2). These findings suggest that EGCG may exert its chemopreventive and anticancer properties, at least in part, by improving potential antitumor immune responses. These findings are significant in the context of chemoprevention and therapy using EGCG since clinical trials have demonstrated that high expression levels of IDO in cancer tissues are associated with disease progression and poor overall survival rates of patients with CRC $(7,8)$.

Efficient EGCG inhibition of IFN- $\gamma$-induced IDO expression in CRC cells may be explained by the inhibition of STAT1 phosphorylation as IFN- $\gamma / \mathrm{JAK} / \mathrm{STAT} 1$ signaling plays a critical role in IDO induction (14). Tyrosine phosphorylation of STAT1 by IFN- $\gamma$-induced reactions promotes the binding of STAT1 to two regulatory elements of the IDO gene promoter region, ISRE and GAS $(14,15)$. In the present study, STAT1 phosphorylation (Fig. 3A) and transcriptional activities of ISRE and GAS (Fig. 3B) induced by IFN- $\gamma$ stimulation were markedly suppressed by EGCG treatment. Our findings suggest that EGCG may prevent IFN- $\gamma$-induced expression of IDO at the gene transcription level through suppressing STAT1 activation. These results are consistent with previous studies demonstrating that EGCG efficiently inhibited IFN- $\gamma$-elicited phosphorylation and/or DNA-binding activity of STAT1 in several types of human cancer-derived cell lines $(25,26)$. In addition to cancer cells, EGCG also inhibits IDO expression induced by IFN- $\gamma$ in dendritic cells through the inhibition of STAT1 phosphorylation (24). Therefore, these studies (24-26), together with our findings, indicate that IFN- $\gamma /$ JAK/STAT1 signaling is a critical target of EGCG in the suppression of IDO induction.

In the present study, the expression levels of IDO mRNA were significantly increased by IFN- $\gamma$ stimulation in all CRC cell lines (Fig. 1A). Additionally, the concentration of L-kynurenine, which reflects the enzymatic activity of IDO in culture medium was also increased by SW837 CRC cells that overexpress IDO (Fig. 2C). These findings may be associated with the immune escape of malignant cells that occurs within the tumor and its surrounding microenvironment since tryptophan depletion and production of toxic tryptophan catabolites resulting from IDO activity may inhibit T cell and natural killer cell proliferation (5). The serum kynurenine/tryptophan ratio is significantly increased, while the serum tryptophan level is significantly decreased, in CRC patients when compared to control noncancerous patients (32). Conversely, IDO inhibitors can impede the growth of IDO-expressing tumors through 
the reduction of kynurenine in the microenvironment and enhancement of $\mathrm{T}$ cell functions (33). Therefore, the effects of EGCG on the suppression of L-kynurenine levels increased by IFN- $\gamma$-stimulated human CRC cells (Fig. 2C) may also contribute to the inhibition of growth of IDO-expressing CRC cells and improve immune tolerance caused by IDO.

In this study, we demonstrate that EGCG downregulates the expression and enzymatic activity of IFN- $\gamma$-induced IDO in CRC cells through the inhibition of STAT1 activation. Our findings suggest the possibility that EGCG may exert its anticancer and chemopreventive effects by inhibiting the expression and function of IDO. A recent experiment in rats has demonstrated that upregulation of IDO activity is possibly involved in colon carcinogenesis, and EGCG treatment effectively suppresses the development of chemically induced colonic preneoplastic lesions by inhibiting the expression levels and enzymatic activity of IDO (12). In conclusion, targeting IDO and improving IDO-mediated immune escape of premalignant and cancer cells with EGCG may be an effective strategy for the prevention and treatment of CRC.

\section{Acknowledgements}

The authors thank Mitsui Norin Co. for providing EGCG.

\section{References}

1. Zitvogel L, Tesniere A and Kroemer G: Cancer despite immunosurveillance: immunoselection and immunosubversion. Nat Rev Immunol 6: 715-727, 2006.

2. Boon $T$ and van der Bruggen P: Human tumor antigens recognized by T lymphocytes. J Exp Med 183: 725-729, 1996.

3. Mellor AL and Munn DH: IDO expression by dendritic cells: tolerance and tryptophan catabolism. Nat Rev Immunol 4: 762-774, 2004

4. Munn DH, Sharma MD, Lee JR, et al: Potential regulatory function of human dendritic cells expressing indoleamine 2,3-dioxygenase. Science 297: 1867-1870, 2002.

5. Frumento G, Rotondo R, Tonetti M, Damonte G, Benatti U and Ferrara GB: Tryptophan-derived catabolites are responsible for inhibition of $\mathrm{T}$ and natural killer cell proliferation induced by indoleamine 2,3-dioxygenase. J Exp Med 196: 459-468, 2002.

6. Lob S, Konigsrainer A, Rammensee HG, Opelz G and Terness P Inhibitors of indoleamine-2,3-dioxygenase for cancer therapy: can we see the wood for the trees? Nat Rev Cancer 9: 445-452, 2009.

7. Brandacher G, Perathoner A, Ladurner R, et al: Prognostic value of indoleamine 2,3-dioxygenase expression in colorectal cancer: effect on tumor-infiltrating T cells. Clin Cancer Res 12: $1144-1151,2006$

8. Ferdinande L, Decaestecker C, Verset L, et al: Clinicopathological significance of indoleamine 2,3-dioxygenase 1 expression in colorectal cancer. Br J Cancer 106: 141-147, 2012.

9. Ninomiya S, Hara T, Tsurumi H, et al: Indoleamine 2,3-dioxygenase in tumor tissue indicates prognosis in patients with diffuse large B-cell lymphoma treated with R-CHOP. Ann Hematol 90: 409-416, 2010.

10. Yoshikawa T, Hara T, Tsurumi H, et al: Serum concentration of L-kynurenine predicts the clinical outcome of patients with diffuse large B-cell lymphoma treated with R-CHOP. Eur J Haematol 84: 304-309, 2010.

11. Ninomiya S, Hara T, Tsurumi H, et al: Indoleamine 2,3-dioxygenase expression and serum kynurenine concentrations in patients with diffuse large B-cell lymphoma. Leuk Lymphoma 53: $1143-1145,2012$

12. Ogawa K, Hara T, Shimizu M, et al: Suppression of azoxymethane-induced colonic preneoplastic lesions in rats by 1-methyltryptophan, an inhibitor of indoleamine 2,3-dioxygenase. Cancer Sci 103: 951-958, 2012.

13. Ikeda $\mathrm{H}$, Old LJ and Schreiber RD: The roles of IFN gamma in protection against tumor development and cancer immunoediting. Cytokine Growth Factor Rev 13: 95-109, 2002.
14. Katz JB, Muller AJ and Prendergast GC: Indoleamine 2,3-dioxygenase in T-cell tolerance and tumoral immune escape. Immunol Rev 222: 206-221, 2008.

15. Chon SY, Hassanain HH and Gupta SL: Cooperative role of interferon regulatory factor 1 and p91 (STAT1) response elements in interferon-gamma-inducible expression of human indoleamine 2,3-dioxygenase gene. J Biol Chem 271: 17247-17252, 1996.

16. Jiang GM, He YW, Fang R, et al: Sodium butyrate down-regulation of indoleamine 2,3-dioxygenase at the transcriptional and post-transcriptional levels. Int J Biochem Cell Biol 42: 1840-1846, 2010.

17. Yang CS, Maliakal $P$ and Meng X: Inhibition of carcinogenesis by tea. Annu Rev Pharmacol Toxicol 42: 25-54, 2002.

18. Yang CS, Wang X, Lu G and Picinich SC: Cancer prevention by tea: animal studies, molecular mechanisms and human relevance. Nat Rev Cancer 9: 429-439, 2009.

19. Shimizu M, Deguchi A, Lim JT, Moriwaki H, Kopelovich L and Weinstein IB: (-)-Epigallocatechin gallate and polyphenon E inhibit growth and activation of the epidermal growth factor receptor and human epidermal growth factor receptor-2 signaling pathways in human colon cancer cells. Clin Cancer Res 11: 2735-2746, 2005.

20. Shimizu M, Deguchi A, Joe AK, McKoy JF, Moriwaki H and Weinstein IB: EGCG inhibits activation of HER3 and expression of cyclooxygenase-2 in human colon cancer cells. J Exp Ther Oncol 5: 69-78, 2005.

21. Shimizu M, Deguchi A, Hara Y, Moriwaki H and Weinstein IB: EGCG inhibits activation of the insulin-like growth factor-1 receptor in human colon cancer cells. Biochem Biophys Res Commun 334: 947-953, 2005

22. Shirakami Y, Shimizu M, Tsurumi H, Hara Y, Tanaka T and Moriwaki H: EGCG and polyphenon E attenuate inflammationrelated mouse colon carcinogenesis induced by AOM and DSS. Mol Med Report 1: 355-361, 2008.

23. Shimizu M, Shirakami Y, Sakai H, et al: (-)-Epigallocatechin gallate suppresses azoxymethane-induced colonic premalignant lesions in male C57BL/KsJ-db/db mice. Cancer Prev Res (Phila) 1: 298-304, 2008.

24. Jeong YI, Jung ID, Lee JS, Lee CM, Lee JD and Park YM: (-)-Epigallocatechin gallate suppresses indoleamine 2,3-dioxygenase expression in murine dendritic cells: evidences for the COX-2 and STAT1 as potential targets. Biochem Biophys Res Commun 354: 1004-1009, 2007.

25. Cheng CW, Shieh PC,Lin YC, et al: Indoleamine 2,3-dioxygenase, an immunomodulatory protein, is suppressed by (-)-epigallocatechin-3-gallate via blocking of gamma-interferon-induced JAK-PKC-delta-STAT1 signaling in human oral cancer cells. J Agric Food Chem 58: 887-894, 2010.

26. Menegazzi M, Tedeschi E, Dussin D, et al: Anti-interferon gamma action of epigallocatechin-3-gallate mediated by specific inhibition of STAT1 activation. FASEB J 15: 1309-1311, 2001.

27. Hoshi M, Saito K, Hara A, et al: The absence of IDO upregulates type I IFN production, resulting in suppression of viral replication in the retrovirus-infected mouse. J Immunol 185: 3305-3312, 2010.

28. Shirakami Y, Shimizu M, Adachi S, et al: (-)-Epigallocatechin gallate suppresses the growth of human hepatocellular carcinoma cells by inhibiting activation of the vascular endothelial growth factor-vascular endothelial growth factor receptor axis. Cancer Sci 100: 1957-1962, 2009.

29. Fujigaki S, Saito K, Sekikawa K, et al: Lipopolysaccharide induction of indoleamine 2,3-dioxygenase is mediated dominantly by an IFN-gamma-independent mechanism. Eur J Immunol 31: 2313-2318, 2001.

30. Muller AJ, DuHadaway JB, Donover PS, Sutanto-Ward E and Prendergast GC: Inhibition of indoleamine 2,3-dioxygenase, an immunoregulatory target of the cancer suppression gene Bin1, potentiates cancer chemotherapy. Nat Med 11: 312-319, 2005.

31. Hou DY, Muller AJ, Sharma MD, et al: Inhibition of indoleamine 2,3-dioxygenase in dendritic cells by stereoisomers of 1-methyltryptophan correlates with antitumor responses. Cancer Res 67: 792-801, 2007.

32. Huang A, Fuchs D, Widner B, Glover C, Henderson DC and Allen-Mersh TG: Serum tryptophan decrease correlates with immune activation and impaired quality of life in colorectal cancer. Br J Cancer 86: 1691-1696, 2002.

33. Koblish HK, Hansbury MJ, Bowman KJ, et al: Hydroxyamidine inhibitors of indoleamine-2,3-dioxygenase potently suppress systemic tryptophan catabolism and the growth of IDO-expressing tumors. Mol Cancer Ther 9: 489-498, 2010. 